\title{
Transformation and detwinning induced electrical resistance variations in $\mathrm{NiTiCu}$
}

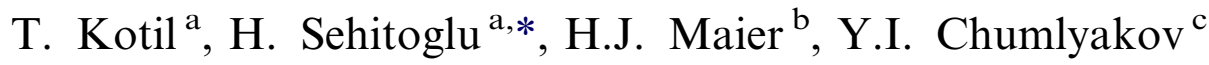 \\ ${ }^{a}$ Department of Mechanical and Industrial Engineering, 1206 West Green Street, University of Illinois, Urbana, IL, 61801, USA \\ ${ }^{\mathrm{b}}$ University of Paderborn, Lehrstuhl für Werkstoffkunde, 33095 Paderborn, Germany \\ ${ }^{\mathrm{c}}$ Siberian Physical and Technical Institute, 634050 Tomsk, Russia
}

Received 14 October 2002

\begin{abstract}
Utilizing electrical resistance measurements in combination with strain observations on single crystals, it was possible to distinguish the austenite to martensite transformation from the detwinning of the martensite behavior during deformation of $\mathrm{NiTiCu}$ shape memory alloys. It was found that the electrical resistance change associated with the detwinning of the martensite variants was as high as $45 \%$ in deformation experiments below the martensite start temperatures. When the conditions led to partial detwinning of the martensite variants (tension at $20{ }^{\circ} \mathrm{C}$ ) the corresponding electrical resistance changes were $20 \%$. In the tension case at $20{ }^{\circ} \mathrm{C}$, the electrical resistance measurements convincingly identified the end of the stress plateau as the conclusion of the transformation and the onset of detwinning. The detwinning is also activated following austenite to martensite transformation under thermal cycling experiments under a constant stress (shape memory under constant stress). In this case, the electrical resistance change associated with detwinning also reached high levels $(45 \%)$ and coincided with the change in slope of the strain-temperature curves.
\end{abstract}

(C) 2003 Elsevier B.V. All rights reserved.

Keywords: $\mathrm{NiTiCu}$; Shape memory; Martensite; Electrical resistance; Detwinning

\section{Introduction}

There is considerable interest in new applications of $\mathrm{NiTiCu}$ alloys which exhibit shape memory effects and pseudoelasticity associated with phase transformation. These materials exhibit a narrower hysteresis compared to their NiTi counterparts [1,2], more stable transformation temperatures after mechanical deformation, and the absence of the R-phase. Depending on the application, they may be deformed in the fully martensitic state, in the fully austenitic state or combination of two to produce different behaviors. In many of the applications, the activation of a transformation cycle is achieved with application of an electric current, therefore the variations in electrical resistance during transformation, martensite rearrangement, and slip of

\footnotetext{
* Corresponding author. Tel.: +1-217-333-4112; fax: +1-217-2446534.

E-mail address: huseyin@uiuc.edu (H. Sehitoglu).
}

$\mathrm{NiTiCu}$ should be fully understood. The knowledge of electrical resistance variations during these sequences can also be utilized to develop an understanding of the underlying microstructural rearrangements.

Electrical resistance measurement has been used in previous work to establish transformation temperatures in a variety of NiTiCu based alloys [3-5]. The electrical resistance changes have been solely attributed to changes in phase transformation. However, the electrical resistance change can be substantial during martensite rearrangement and this has received very little attention. Recently, Pozzi and Airoldi [6] has pointed out that resistivity of the alloy changes during reorientation of the martensite variants. De Araujo et al. [7] also observed electrical resistance variations of nearly $15 \%$ with increasing stress levels and attributed it to the rearrangement of martensite variants. If the magnitude of transformation-induced and martensite rearrangement-induced resistance changes are established this information can be used for a clear interpretation of 
the deformation behavior of shape memory alloys under a variety of thermo-mechanical loading conditions. There is a lack of understanding of the fundamental mechanisms in shape memory alloys and this has resulted in numerous phenomenological models that can only capture limited deformation behaviors.

Our previous work has focused on compression deformation and thermal cycling behavior of $\mathrm{NiTiCu}$ alloys [1,2] without electrical resistance measurements. It was noted that detwinning of the martensite contributed to the higher strains observed in the thermal cycling experiments [2] compared to the mechanical deformation case [1]. The theoretical transformation strain calculations for the transformation and the detwinning strain for the martensite (the growth of one of the variants within a martensite) structure were included for comparison and interpretation of experimental results $[1,2]$. The present study is designed to understand the evolution of phase transformation and the martensite rearrangement (detwinning) in mechanical deformation experiments above and below transformation temperature, and also under constant stress thermal cycling with simultaneous measurements of strain and electrical resistance.

Three single crystal orientations are chosen in the present work ([111], [011], and [012]). The choice of single crystals facilitates the interpretation of experimental results due to absence of grain boundaries and the ability to reach high strains. Majority of the results presented will be on [012] single crystals as the transformation proceeds through nearly a single step (cubic to monoclinic) in this case facilitating the interpretation of the results. The choice of NiTiCu (as opposed to NiTi) is preferrred because the measured electrical resistance changes are substantial (as high as 55\%) in $\mathrm{NiTiCu}$ compared to NiTi $(\sim 30 \%)$. The NiTiCu exhibits a continous rise in electrical resistance corresponding to the entire phase transformation and martensite detwinning stages while in $\mathrm{NiTi}$ alloys the electrical resistance exhibits a drop with temperature. The variation in electrical resistance due to transformation is small $(10 \%)$ while it is substantial $(45 \%)$ during martensite rearrangement. In addition to the thermomechanical loading cases, and the electrical resistance measurements, TEM results are included to gain insight into the complex microscopic evolutions.

\section{Material and experimental techniques}

The single crystals of NiTiCu were grown in an inert environment using a Bridgman furnace. The specimens are a dog bone type with an $8 \mathrm{~mm}$ gage length and $1 \times 3$ $\mathrm{mm}$ rectangular gage cross-section. They were prepared by EDM machining. After machining, they were solutionized at $920{ }^{\circ} \mathrm{C}$ for $24 \mathrm{~h}$ in a vacuum furnace and then water quenched. The surface damages due to EDM machining and oxidation during quenching have been removed by mechanical polishing. During the experiments, strain, stress, electrical resistance and temperature were measured simultaneously. A miniature extensometer, with a gage length of $3 \mathrm{~mm}$, was used to measure the axial deformation. During isothermal monotonic tensile testing, a very low strain rate was chosen, $10^{-4} 1 \mathrm{~s}^{-1}$, to minimize temperature rise. Similarly, unloading was performed with approximately the same strain rate.

The transformation temperatures for the solutionized state have been measured by differential scanning calorimetry (DSC) (Fig. 1). The experiment was conducted on Perkin-Elmer Pyris I DSC machine with $10{ }^{\circ} \mathrm{C} \min ^{-1}$ temperature rate. The austenite and martensite start and finish temperatures are $A_{\mathrm{s}}=0{ }^{\circ} \mathrm{C}$, $A_{\mathrm{f}}=31{ }^{\circ} \mathrm{C}, M_{\mathrm{s}}=21{ }^{\circ} \mathrm{C}, M_{\mathrm{f}}=-11{ }^{\circ} \mathrm{C}$, respectively. It is noted that the transformation temperatures from DSC peaks indicate the first stage of transformation (B2-B19). The successive stage $\left(\mathrm{B} 19-\mathrm{B} 19^{\prime}\right)$ occurs at slightly lower temperatures but is not detected with the DSC. We will not make a demarcation between the two stages as this has been addressed in previous studies. The focus will be placed on the rearrangements in martensite during deformation below transformation temperatures or following phase transformation.

Deformation experiments were carried out on $\mathrm{Ti}-$ $40 \mathrm{Ni}-10 \mathrm{Cu}$ single crystal shape memory alloys at different temperatures. The results are demonstrated for the crystallographic orientations ([012] but similar results have been obtained for other orientations ([111], [011]). The theoretical transformation strains for the [012] case are 5.42\% for CVP ( correspondent variant pair) formation and $6.32 \%$ including detwinning of the B19'. Strain was controlled during loading and stress was controlled during unloading to zero stress. The maximum strain was chosen as $5 \%$, which is high enough to cause both martensite transformation and detwinning, but small enough to avoid gross plastic deformation at -60 and $20{ }^{\circ} \mathrm{C}$. Three different testing temperatures were chosen; $-60,20$ and $100{ }^{\circ} \mathrm{C}$. Every tensile test was followed by a heating cycle. Strainelectrical resistance measurements were used to monitor the recovery. Zero load level was chosen in order to prevent any plastic deformation during heating. Samples that contained martensite after deformation were heated to recover the transformation strains. Maximum temperature for heating was $60{ }^{\circ} \mathrm{C}$, which is well above the $A_{\mathrm{f}}$ temperature. Above this temperature no additional recovery was observed.

In a second series of experiments temperature cycling under constant stress was conducted between 100 and $-100{ }^{\circ} \mathrm{C}$. This type of experiment is sometimes referred to as 'stress-assisted two way shape memory effect'. In these experiments, the stress level is chosen high enough 


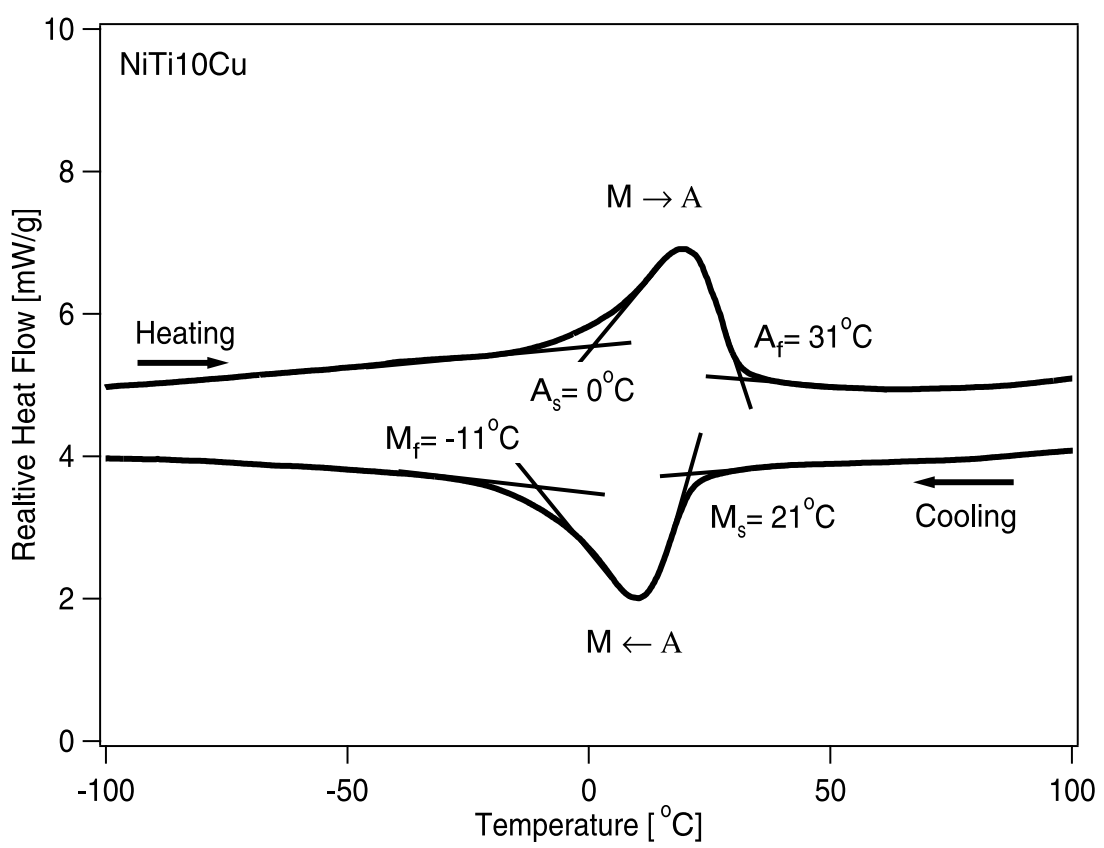

Fig. 1. The transformation temperatures are determined by differential scanning calorimetry (DSC) study. The austenite and martensite start and finish temperatures are $A_{\mathrm{s}}=-0{ }^{\circ} \mathrm{C}, A_{\mathrm{f}}=31{ }^{\circ} \mathrm{C}, M_{\mathrm{s}}=21{ }^{\circ} \mathrm{C}, M_{\mathrm{f}}=-11{ }^{\circ} \mathrm{C}$, respectively.

to induce transformation upon heating and cooling but low enough to minimize plastic deformation. This type of experiment, besides revealing fundamental information on how the transformation temperatures are affected by stress, is extremely useful in understanding the behavior of SMA actuators.

A Tinsley Senator type 5840D AC strain meter was used for the electrical resistance measurements. The electrical resistance measurement range was $100 \Omega$ with $\pm 1 \mu \Omega$ accuracy. The 4-wire lead measurement method was used, which cancels lead resistance errors inherent in 2-wire systems. By twisting the four lead wires, this technique was very effective to reduce the electrical noise, which could originate from the induction generator used to heat the samples in a loading stage. The measured electrical resistance variation is due to phase transformation and martensite rearrangement (detwinning), temperature induced changes, plastic deformation and specimen geometry (strain) changes. A normalized electrical resistance, $\Delta R / R$, is defined by removing the temperature and geometry effects from the measured electrical resistance. The correction to the measured electrical resistance, $\Delta \rho / \rho$, is made as follows,

$\frac{\Delta R}{R}=\frac{\Delta \rho}{\rho}-(1+2 v) \varepsilon-C\left(T-T_{0}\right)$

where $\left.(1+2 v) \varepsilon, C\left(T-T_{0}\right)\right)$ are terms corresponding to the change in relative electrical resistance due to geometry (shape) change and temperature respectively. The constant $C$ in Eq. (1) accounts for change in resistivity with temperature and is determined from the slope of the normalized resistance-temperature curve in the absence of transformation. The value of $C$ is $-2.1 \times 10^{-4} 1 /{ }^{\circ} \mathrm{C}$. The $T_{0}$ is reference temperature and is taken as $100{ }^{\circ} \mathrm{C}$ in this study. The effect of plastic deformation was negligible $(<2 \%)$ based on the measurements at $100{ }^{\circ} \mathrm{C}$, therefore a plastic strain induced correction is not required in Eq. (1).

\section{Results}

With three sets of experiments, tensile loading below the martensite finish temperature, and thermal cycling under zero and constant stress, we demonstrate that the normalized electrical resistance measurement could be effectively utilized to separate martensite formation from detwinning. The normalized electrical resistance change due to martensite to austenite transformation was $\sim 0.1$ while for the case of detwinning the electrical resistance change was as high as 0.45 .

In Fig. 2(a) and (b) the stress-strain and normalized electrical resistance-strain behavior are shown for tensile loading at $100,-60$ and $20{ }^{\circ} \mathrm{C}$. The test temperature of $100{ }^{\circ} \mathrm{C}$ is above the $M_{\mathrm{d}}$ temperature, which is the highest temperature for stress induced transformation. At $100{ }^{\circ} \mathrm{C}$ the electrical resistance variation was near $2 \%$, which is attributed to plastic deformation in the austenite domains.

We first describe the tensile experiment at $-60{ }^{\circ} \mathrm{C}$ in Fig. 2(a) and (b) and in more detail in Fig. 3(a) and (b). This temperature is much lower than the $M_{\mathrm{f}}$, martensite finish temperature. The deformation occurs via detwinning of the martensite variants known as correspondent 

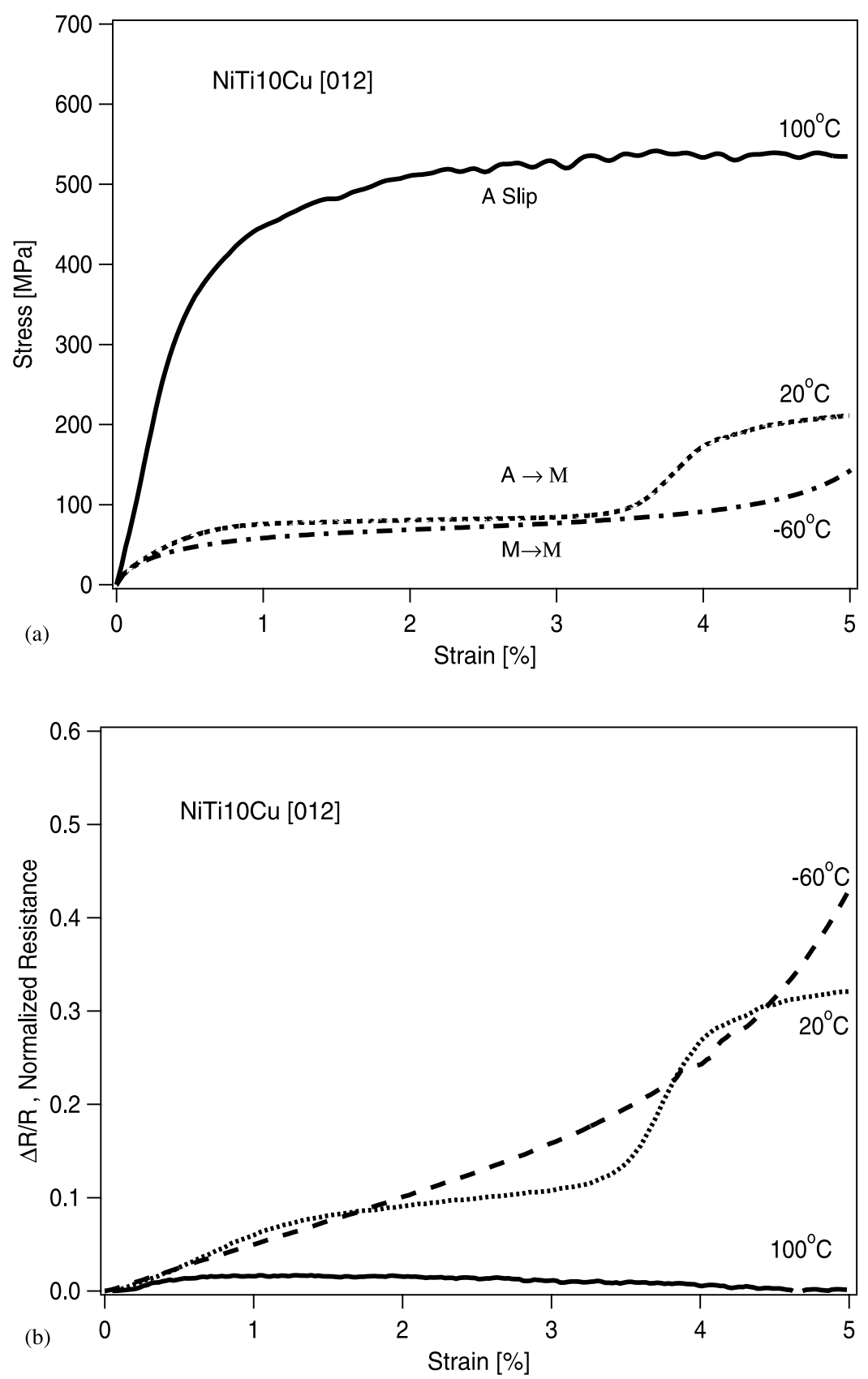

Fig. 2. (a) Tensile stress-strain and (b) normalized electrical resistance-strain behavior of single crystal NiTi10Cu [012] orientation. Testing temperatures are 100,20 and $-60{ }^{\circ} \mathrm{C}$.

variant pairs (CVPs). The upward curvature at high strains at $-60{ }^{\circ} \mathrm{C}$ corresponds to the elastic deformation of the detwinned martensite (Figs. 2(a), and 3(a)). In Fig. 3(a) the measured normalized electrical resistance change (dashed line) is superimposed on the stress-strain curve (solid line). Based on these results the normalized electrical resistance change $\left(\mathrm{OA}^{\prime}\right)$ due to the detwinning is rather substantial, $\sim 45 \%$. During unloading to zero stress, elastic deformation and partial reverse detwinning were observed $\left(\mathrm{AB}\right.$ and $\mathrm{A}^{\prime} \mathrm{B}^{\prime}$ in Fig. 3(a) and (b)). A heating cycle followed at zero stress, where the specimen was heated from -60 to $+60{ }^{\circ} \mathrm{C}$ and then cooled to $-60{ }^{\circ} \mathrm{C}$ before the next cycle was started. The strain-temperature (solid line) path follows $\mathrm{BCO}$ and electrical resistance-temperature (dashed line) curves follow $\mathrm{B}^{\prime} \mathrm{C}^{\prime} \mathrm{D}^{\prime} \mathrm{O}^{\prime}$ in Fig. 3(a). The strain and electrical resistance are plotted versus temperature in Fig. 3(b) during heating at zero stress to gain further insight into results. During initial heating detwinning occurs and normalized resistance changes by $44 \%$. Then, a substantial change in slope occurs at a temperature of $12{ }^{\circ} \mathrm{C}$, which marks the demarcation between transformation and detwinning. The reverse detwinning occurred during $\mathrm{A}^{\prime} \mathrm{B}^{\prime} \mathrm{C}^{\prime}$ or $\mathrm{ABC}$ and is followed by the 

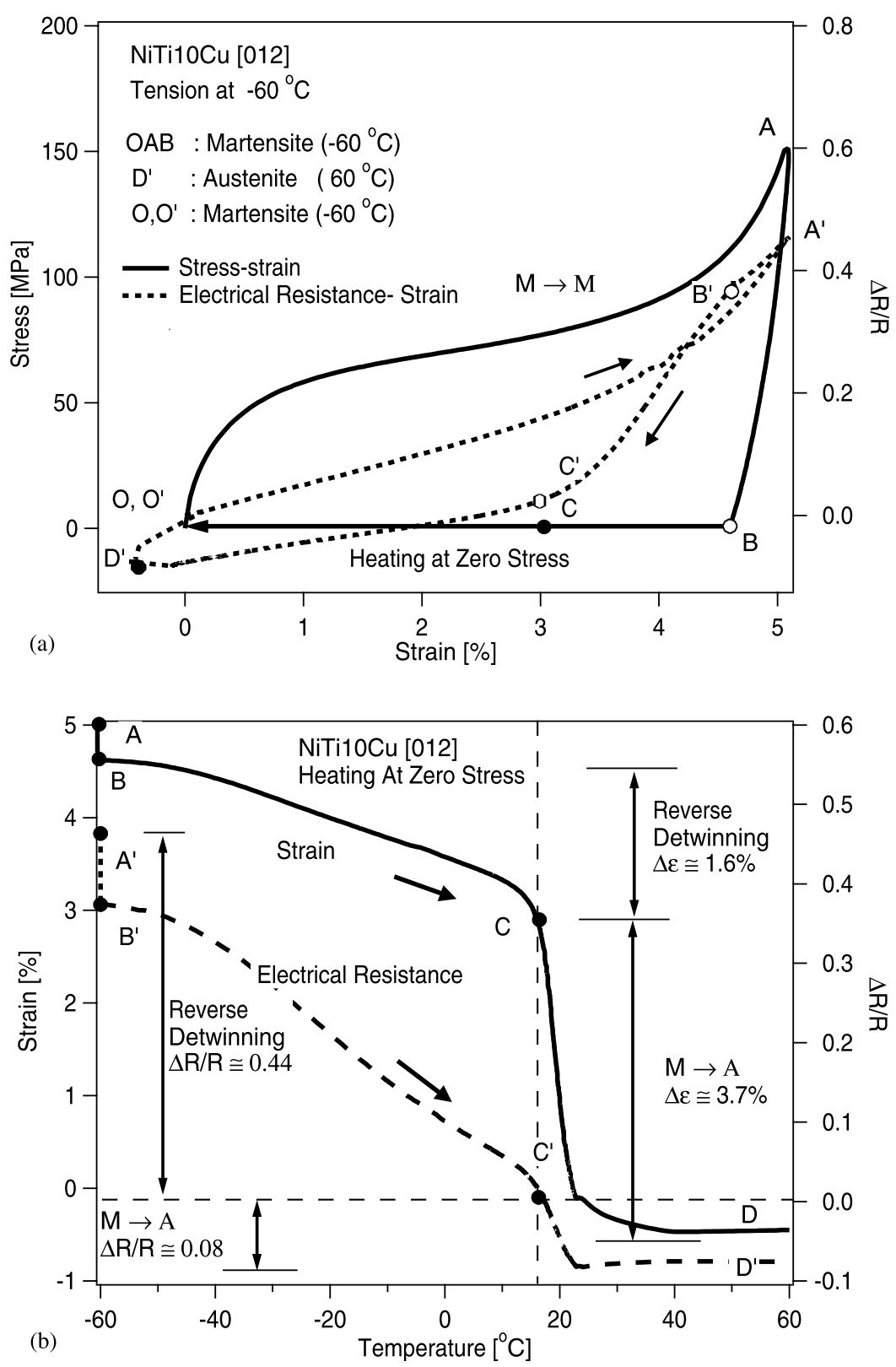

Fig. 3. (a) Tensile stress-strain and normalized electrical resistance-strain behavior of single crystal NiTi10Cu [012] orientation. Testing temperature is $-60{ }^{\circ} \mathrm{C}$, (b) heating behavior $(\sigma=0 \mathrm{MPa})$ displaying the strain normalized electrical resistance recoverability.

martensite to austenite transformation $\left(\mathrm{C}^{\prime} \mathrm{D}^{\prime}\right)$. During segment $\mathrm{D}^{\prime} \mathrm{O}^{\prime}$ in Fig. 3(a) the specimen was cooled to $-60{ }^{\circ} \mathrm{C}$. The difference between $\mathrm{O}^{\prime}$ and $\mathrm{D}^{\prime}$ corresponds to the electrical resistance change under stress free cycling conditions of $\sim 10 \%$. We did not include the cooling portion of the test (after $\mathrm{D}^{\prime}$ ) in Fig. 3(b) to reduce the complexity in the graphs.

The shift in $A_{\mathrm{s}}$ temperature $\left(\Delta A_{\mathrm{s}} \approx 12{ }^{\circ} \mathrm{C}\right)$ is expected in view of the prior deformation. Under stress free cycling the $A_{\mathrm{s}}$ temperature is $0{ }^{\circ} \mathrm{C}$ from the DSC results (Fig. 1). The SME strains corresponding to detwinning and transformation in Fig. 3(b) are 1.6 and 3.7\%, respectively.
We next show the strain-temperature and electrical resistance-temperature variations from the thermal cycling test under constant stress in Fig. 4(a). In this experiment the specimen was cooled from 100 to $-100{ }^{\circ} \mathrm{C}$ and then heated from -100 back to $100{ }^{\circ} \mathrm{C}$ under a constant stress of $125 \mathrm{MPa}$. The change in slope of the strain-temperature curve occurs at point $\mathrm{C}$ with a corresponding point of $\mathrm{C}^{\prime}$ in the electrical resistance temperature curve. Remarkably, the electrical resistance change of $\mathrm{A}^{\prime} \mathrm{C}^{\prime}$ corresponds to 0.44 . This change is identical to the measured change in Fig. 3(a) under detwinning. The austenite to martensite transformation regime is marked on the figure as $\mathrm{C}^{\prime} \mathrm{D}^{\prime}$ and corresponds 

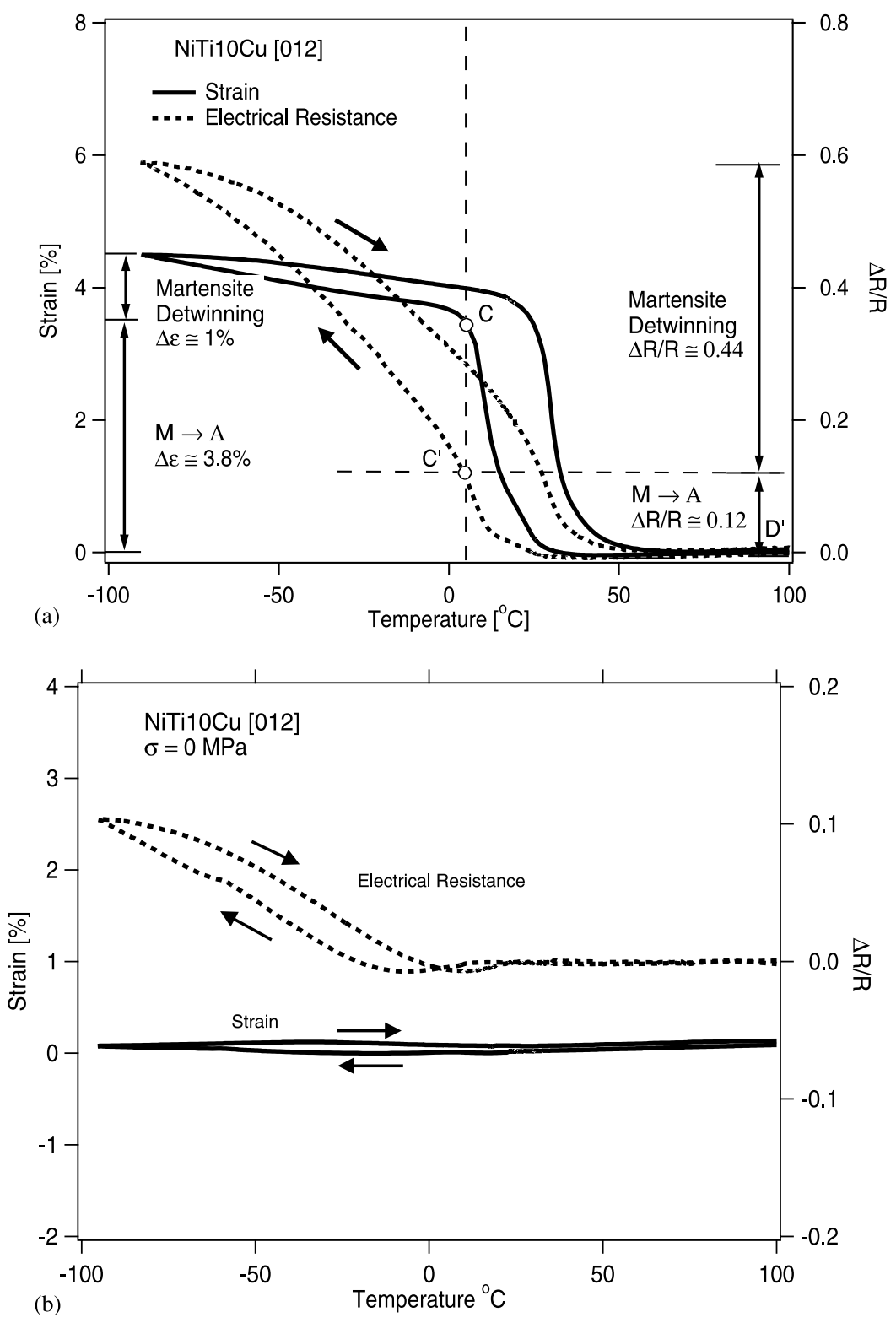

Fig. 4. (a) Strain-temperature and electrical resistance-temperature variation during constant stress ( $=125 \mathrm{MPa})$ thermal cycle test of NiTi10Cu [012], (b) strain and resistance variations under thermal cyling with zero stress, (c) Thermal cycling of [111] and [012] under a constant stresss of 175 $\mathrm{MPa}$.

to $12 \%$. The strain changes due to transformation and detwinning were 3.8 and $1.0 \%$, respectively, and are also marked on the figure.

To gain further insight into results, the normalized electrical resistance and measured strain are plotted versus temperature in Fig. 4(b) for zero stress thermal cycling. We note that in the absence of applied stress the measured strains are nearly zero. The maximum change in normalized electrical resistance approaches 0.1 . Note that the temperature induced resistance change is subtracted from the measured resistance change in this case. The normalized resistance change of 0.1 (or $10 \%$ ) associated with the entire transformation is consistent with the previous studies of Nam et al. [5].
The experimental results of strain-temperature for [111] and [012] cases are compared in Fig. 4(c). It is noted that the [111] case produces the highest strains and the highest electrical resistance changes. The maximum strain levels are 6.5 and $5 \%$ for the [111] and [012] cases, respectively. For the [012] case we note the presence of a residual strain of $0.3 \%$ at the conclusion of the thermal cycle. This is indicative of the high stress levels (175 MPa) triggering plastic deformation in the austenite domains.

Finally we consider in detail the results of the tensile loading -unloading -heating at zero stress experiment at $20{ }^{\circ} \mathrm{C}$ in Fig. 5(a). We note that the stress scale in this case is different from Fig. 3(a). The location $\mathrm{P}^{\prime}$ 


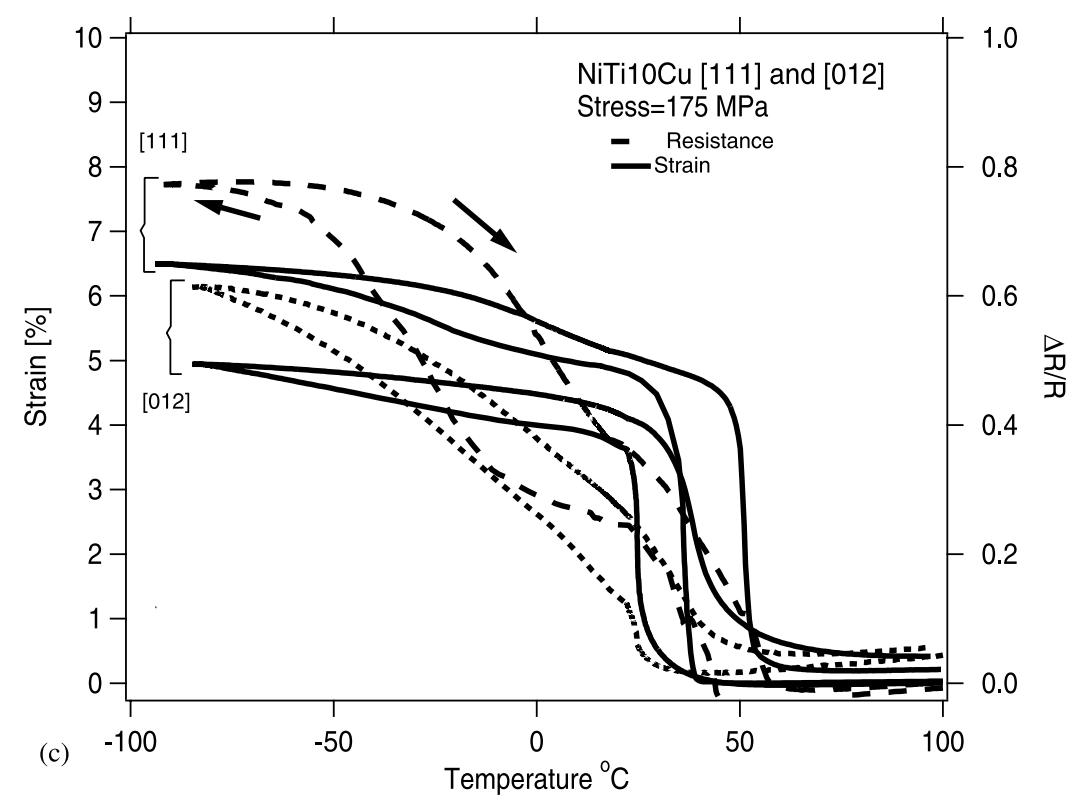

Fig. 4 (Continued)

corresponding to a normalized electrical resistance change of $13 \%$ also corresponds to the end of the plateau in the stress-strain curve (point $\mathrm{P}$ ). This is followed by martensite detwinning (PA and $\left.\mathrm{P}^{\prime} \mathrm{A}^{\prime}\right)$ where a marked increase in normalized electrical resistance slope appears as the strains approach 5\%. The change in normalized electrical resistance is $19 \%$ which indicates that partial detwinning of the martensite has occurred. During unloading only partial reverse detwinning occurs until zero stress is reached (point $\mathrm{B}$ ) corresponding to an electrical resistance change of $17 \%$. A heating cycle was applied under zero stress where the specimen was heated from 20 to $60{ }^{\circ} \mathrm{C}$. Austenite to martensite transformation evolves at zero stress upon heating. The electrical resistance change during this transformation back to martensite is $11 \%$. Similar variations in normalized electrical resistance were obtained in our experiments when the tensile cycle is repeated with slight modification in the shape of the stress-strain curves, so these results are not included here.

To compare the behavior of [011] and [111] orientations the results for tension case are provided in Fig. 5(b). The [111] crystal orientation has the highest resolved shear stress factor and high transformation strains in tension. We note that the corresponding normalized electrical resistance changes are nearly 0.35 in the [111] case and 0.25 in the [011] case. The trends are similar to the details given in Fig. 5(a). The locations corresponding to the conclusion of the transformation and the onset of martensite rearrangement are marked on the figure with a dot.

Electron microscopy has proved valuable to identify the microstructural changes in $\mathrm{NiTiCu}$ alloys and only the representative features are given here. The features of martensites in NiTiCu are submicron dimensions and can not be ascertained with optical microscopy techniques. The presence of internal twinning in the martensites is highlighted in Fig. 6(a) and (b). These results were obtained from mechanical deformation experiments illustrated in Fig. 5(a) for the [012] case. Several observations are noteworthy. Some martensite is stabilized after cyclic deformation. This means that after heating at zero stress residual martensite is present explaining the nonrecoverability of the strain in the tension case shown in Fig. 5(a). The martensite is internally twinned and consists of two variants with one variant occupying a lower volume fraction than the other.

Stabilized martensite after thermal cycling is illustrated in Fig. 7 showing a group of variants which are internally twinned and one which is detwinned (left hand side). The presence of the underlying dislocation structure is also visible in this figure. In the [111] case the presence of substantial dislocation activity will ultimately limit the transformation strain from attaining theoretical values.

\section{Discussion}

The purpose of the paper has been to understand the mechanisms of phase transformation and martensite rearrangement (internal detwinning) in solutionized $\mathrm{NiTiCu}$ alloys. With combination of electrical resistance measurements, electron microscopy results and strain measurements, it was possible to identify the onset of transformation and detwinning under both shape memory and pseudoelasticity conditions. Based on these 

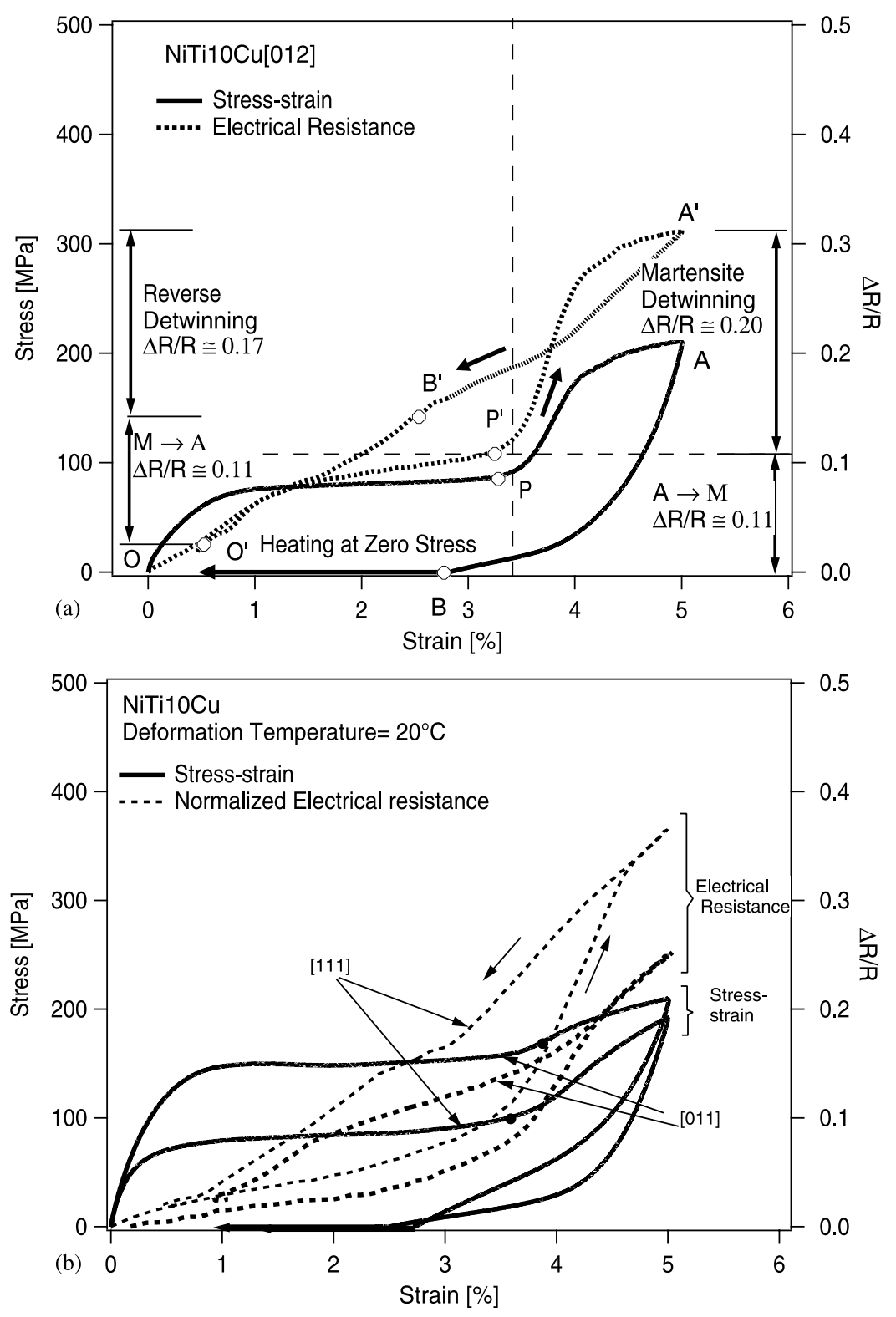

Fig. 5. (a) Cyclic stress-strain and electrical resistance-strain behavior of single crystal NiTi10Cu shape memory alloys at [012] orientation. (b) Cyclic stress-strain and electrical resistance-strain behavior of single crystal NiTi10Cu shape memory alloys at [012] orientation. The dots on the solid line mark the onset of martensite rearrangement.

observations, modeling efforts could be better channeled to incorporate physical mechanisms of deformation in NiTiCu alloys.

The experimental results from selected crystal orientations confirm that $\mathrm{NiTiCu}$ alloys are capable of achieving large recoverable strains (as high as 6.5\%) with large increases in electrical resistance. The results point out that these alloys exhibit narrow hysteresis and near [111] orientations display the largest strain compared to other orientations. The strain results fall short of the theoretical values because of inelastic flow causing permanent deformation. The previous works utilizing polycrystals [3,5] have shown that the transformation strains are near $3.5 \%$ which is substantially lower than those observed in this work.

The experiments revealed that large strains are recoverable without transformation $(\mathrm{M} \rightarrow \mathrm{M})$ and with phase transformation $(\mathrm{A} \rightarrow \mathrm{M})$ in $\mathrm{NiTiCu}$ alloys. The degree of recoverability is higher for the $\mathrm{M} \rightarrow \mathrm{M}$ case based on the strain measurements and electrical resistance. The presence of residual martensite and strong dislocation activity, as observed from electron microscopy, is responsible for partial loss of shape memory in mechanical deformation above the transition temperatures and also under thermal cycling under stress. The residual martensite, which is often pinned by lattice 


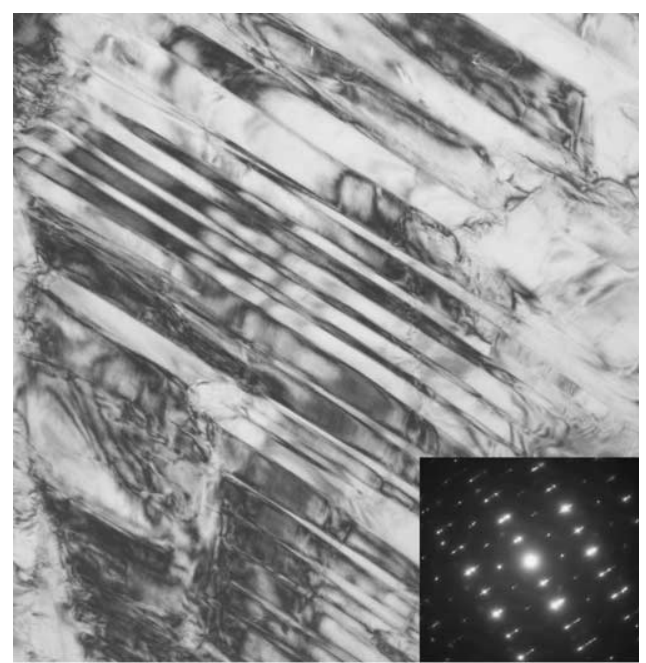

(a)

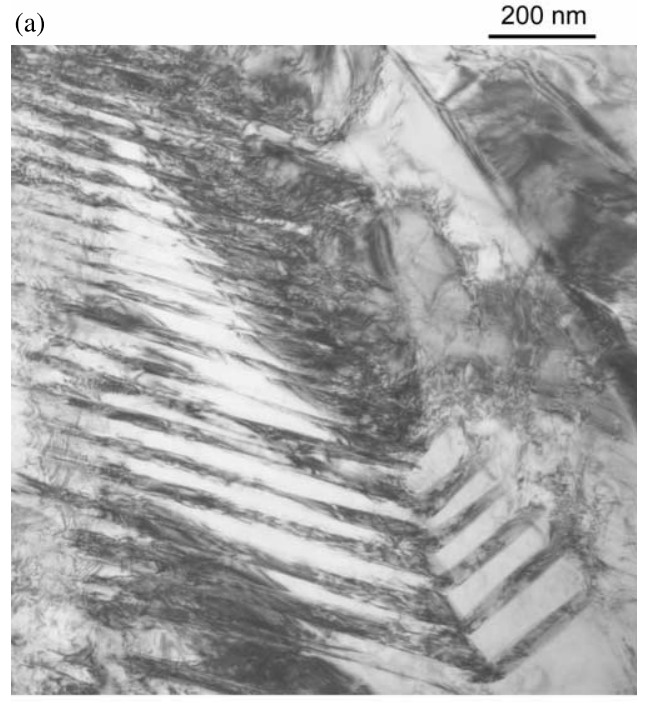

(b)

$500 \mathrm{~nm}$

Fig. 6. TEM images from the [012] NiTiCu subjected to mechanical deformation experiments to $5 \%$ strain, (a) martensite variants with a diffraction pattern showing that the variants are twin related, (b) internal twinning in martensite domains also showing dislocation activity and martensite variants.

defects such as dislocations, limits the volume of austenite domains available for transformation.

In addition to the electrical resistance measurements under stress, the normalized electrical resistance levels were measured under zero stress and the corresponding change was determined as $10 \%$. This result is consistent with the work of Nam et al. [5] who also made similar measurements. The onset of transformation in Fig. 4(b) is consistent with the DSC results and represents the B19 start temperature. The separation of martensite start temperatures between B19 and B19' is rather small (a few degrees) and a second peak is not discernable from the results. For all practical purposes, it is difficult to separate these two transformations because in mechan-

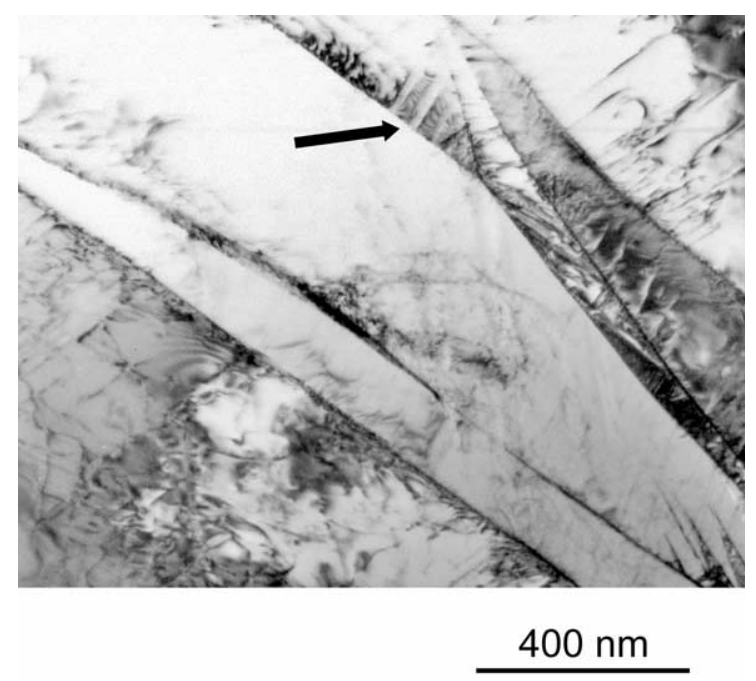

Fig. 7. A TEM image from thermal cycling of the NiTiCu [111] sample under a constant stress of $125 \mathrm{MPa}$. A group of martensite variants are shown with an internally twinned structure (arrow) and one variant (on the left hand side) which has detwinned.

ical deformation and thermal cycling a steep change in the stress-strain response can not be detected and the second transformation follows almost simultaneously. The normalized resistance change of 0.1 corresponding to the transformation is considerably smaller than the resistance change levels observed in mechanical deformation and thermal cycling experiments (0.6). Since the B19 is essentially twinless, it is the detwinning of the B19' that produces substantial changes in mechanical and electrical properties. It is also noted that the measured strains in the zero stress thermal cycling case is nearly zero and this is consistent with the self accommodating nature of the martensite variants forming energetically favorable groupings resulting in a net zero strain. Under stress, the detwinning induced strain is of the order of $1.5 \%$ (in tension) with an associated resistance change as high as $45 \%$. The calculated values of strain between experiment and theory agree on the magnitude of the strains [2].

A novel finding in this work is that the electrical resistance change during rearrangement of martensite is substantial and large strains are recoverable in $\mathrm{NiTiCu}$ alloys. The transformation strain in NiTiCu alloys is limited by the activation of slip. The amount of slip irreversibility is crystal orientation dependent and depends on the operative slip systems in the particular crystal orientation. It is well known that $\langle 100\rangle\{001\}$ and $\langle 100\rangle\{011\}$ slip sytems are operative in these alloys and with the exception of the crystal orientations near the [001] pole other orientations have a finite Schmid factor. Based on the Schmid factors for plastic deformation both the [111] and [001] orientations are unfavorably oriented. Consequently, the formation of inelastic strain during thermal cycling bounds the transformation 
strain. The inelastic strain occurs when the applied stress exceeds the critical transformation stress. Further heat treatments are expected to increase the flow resistance of austenite domains and allow the experimental transformation strains to approach the theoretical values.

\section{Conclusions}

1. The normalized electrical resistivity in $\mathrm{NiTiCu}$ alloys exhibits a distinct increase over the entire transformation and the martensite rearrangement stages. The changes in electrical resistance is considerably larger (as high as 60\%) compared to the natural resistivity changes in the absence of microstructural changes.

2. The electrical resistance measurements were utilized to separate the martensite transformation from rearrangement of martensite (detwinning) in an $\mathrm{NiTiCu}$ alloy under both temperature cycling and isothermal deformation experiments. The electrical resistance changes due to the martensite transformation and detwinning were 10 and $44 \%$, respectively. When partial detwinning occurred, such as in the tensile experiment conducted at $20{ }^{\circ} \mathrm{C}$, the detwinning portion was limited to $20 \%$.

3. For all three crystal orientations subjected to tensile experiments at $20{ }^{\circ} \mathrm{C}$, the electrical resistance measurements confirmed that the end of the stress plateau region is the conclusion of the austenite to martensite transformation and the onset of detwinning. Reverse detwinning dominates the pseudoleastic response portions of the stress-strain curve with reverse phase transformation confined to the heating treatment at zero stress.
4. The experiments reveal that full recoverability of strains is restricted in the presence of residual martensite and slip deformation as seen in the electron micrographs. The martensite domains observed with electron microscopy exhibited both detwinned and twinned variants demonstrating that the entire detwinning process has not reached a completion.

\section{Acknowledgements}

This work was partially supported by the Air Force Office of Scientific Research, grant no. F49620-01-10136.

\section{References}

[1] H. Sehitoglu, I. Karaman, X. Zhang, H. Kim, Y. Chumlyakov, H.J. Maier, I. Kireeva, Metall. Mater. Trans. A 32 (3) (2001) 477489.

[2] H. Sehitoglu, I. Karaman, X. Zhang, A. Viswanath, Y. Chumlyakov, H.J. Maier, Acta Mater. 49 (17) (2001) 3621-3634.

[3] W.J. Moberly, K.N. Melton, in: W. Duering, K.N. Melton, D. Stockel, C.M. Wayma (Eds.), Engineering Aspects of Shape Memory Alloys, Butterworth-Heinemann, pp. 46-57.

[4] Yu.I. Chumlyakov, I.V. Kireeva, Yu.I. Zuev, AG. Lyisyuk, Shape memory effect and pseudoelasticity in $\mathrm{Ti}-40 \% \mathrm{Ni}-10 \% \mathrm{Cu}$ (at.\%) single crystals, Materials for Smart Systems II Materials Research Society Symposium Proceedings, vol. 459, Materials Research Society, Pittsburgh, 1997, pp. 401-406.

[5] T.H. Nam, T. Saburi, K. Shimizu, JIM 31 (11) (1990) 959-967.

[6] M. Pozzi, G. Airoldi, Mater. Sci. Eng. A273 (1999) 300-304.

[7] C.J. De Araujo, M. Morin, G. Guenin, Mater. Sci. Eng. A273 (1999) 305-309. 